Bull. Korean Math. Soc. 51 (2014), No. 4, pp. 1075-1085

http://dx.doi.org/10.4134/BKMS.2014.51.4.1075

\title{
ASYMPTOTIC EQUIVALENCE BETWEEN TWO LINEAR DYNAMIC SYSTEMS ON TIME SCALES
}

\author{
Sung Kyu Choi And Namjip Koo
}

\begin{abstract}
In this paper we investigate asymptotic properties about asymptotic equilibrium and asymptotic equivalence for linear dynamic systems on time scales by using the notion of $u_{\infty}$-similarity. Also, we give some examples to illustrate our results.
\end{abstract}

\section{Introduction}

The calculus on time scales was initiated by Aulbach and Hilger in order to create a theory that can unify and extend discrete and continuous analysis $[1,2,15]$. The theory on time scales has been developed as a generalization of both continuous and discrete time theory and applied to many different fields of mathematics $[1,2,3,4]$.

The notion of similarity is an effective tool to study the theory of stability for differential systems and difference systems [5, 7, 10, 11, 12, 17, 18, 19]. Markus [17] introduced the notion of kinematic similarity in the set of all $n \times n$ continuous matrices defined on $\left[t_{0}, \infty\right)$ and showed that the relationship of kinematic similarity is an equivalence relation preserving the type numbers of the linear differential systems. Gohberg et al. [14] studied the problem to classify linear time-varying systems of difference equations under kinematic similarity. Conti [12] introduced the concept of $t_{\infty}$-similarity in the set of all $n \times n$ continuous matrices defined on $\mathbb{R}_{+}=[0, \infty)$ and showed that $t_{\infty^{-}}$ similarity is an equivalence relation preserving strict, uniform and exponential stability of linear homogeneous differential systems. Choi et al. [9] studied the variational stability of nonlinear differential systems using the notion of $t_{\infty}$-similarity. Trench [18] introduced a concept called $t_{\infty}$-quasisimilarity that is not symmetric or transitive, but still preserves stability properties.

As a discrete analog of Conti's definition of $t_{\infty}$-similarity, Trench [19] defined the notion of summable similarity on pairs of $m \times m$ matrix functions and showed that if $A$ and $B$ are summably similar and the linear system $\Delta x(n)=$

Received August 17, 2013.

2010 Mathematics Subject Classification. 34A30, 34D05, 34D10, 34D20, 34N05.

Key words and phrases. asymptotic equivalence, asymptotic equilibrium, $u_{\infty}$-similarity, strong stability, linear dynamic systems, time scales. 
$A(n) x(n), n=0,1, \ldots$, is uniformly, exponentially or strictly stable or has linear asymptotic equilibrium, then the linear system $\Delta y(n)=B(n) y(n)$ has also the same properties. Also, Choi and Koo [7] introduced the notion of $n_{\infty}$-similarity in the set of all $m \times m$ invertible matrices and showed that two concepts of global $h$-stability and global $h$-stability in variation are equivalent by using the concept of $n_{\infty}$-similarity and Lyapunov functions. Their approach included most types of stability.

Choi et al. $[9,10,11]$ investigated asymptotic equivalence for differential systems by means of the notions of strong stability and $t_{\infty}$-similarity introduced by Conti [12]. Trench [19] introduced summable similarity as a discrete analog of Conti's definition of $t_{\infty}$-similarity and investigated the various stabilities of linear difference systems by using summable similarity. Choi et al. [5] studied the asymptotic property and the $h$-stability of difference systems via discrete similarities and comparison principle. For detailed results about the various stabilities including the notions of $h$-stability and strong stability of dynamic systems on time scales, see $[6,8]$.

In this paper we investigate asymptotic properties about asymptotic equilibrium and asymptotic equivalence for linear dynamic systems on time scales by using the notion of $u_{\infty}$-similarity. Also, we give some examples to illustrate our results.

\section{Main results}

We refer the reader to Ref. [3, 4] for all the basic definitions and results on time scales necessary to this work (e.g. delta differentiability, rd-continuity, exponential function and its properties).

Throughout this paper, we assume that the time scale $\mathbb{T}$ (a nonempty closed subset of $\mathbb{R}$ ) is unbounded above and the graininess of $\mathbb{T}$ is bounded on $\mathbb{T}_{t_{0}}$. Here $\mathbb{T}_{t_{0}}=\mathbb{T} \cap\left[t_{0}, \infty\right)$ for each fixed $t_{0} \in \mathbb{T}$. If $\mathbb{T}$ has a left-scattered maximum $m$, then $\mathbb{T}^{\kappa}=\mathbb{T}-\{m\}$. Otherwise, $\mathbb{T}^{\kappa}=\mathbb{T}$. Assume that $\mathbb{R}^{n}$ is the $n$-dimensional real Euclidean space.

Let $M_{n}(\mathbb{R})$ be the set of all $n \times n$ matrices over $\mathbb{R}$ and $\mathfrak{M}_{n}(\mathbb{R})$ the set of all $n \times n$ invertible matrices over $\mathbb{R}$.

Definition 2.1. An operator $A: \mathbb{T}^{\kappa} \rightarrow M_{n}(\mathbb{R})$ is called regressive if for each $t \in \mathbb{T}^{\kappa}$ the $n \times n$ matrix $I+\mu(t) A(t)$ is invertible.

The class of all rd-continuous and regressive operators from $\mathbb{T}^{\kappa}$ to $M_{n}(\mathbb{R})$ is denoted by

$$
C_{\mathrm{rd}} \mathcal{R}\left(\mathbb{T}^{\kappa}, M_{n}(\mathbb{R})\right) .
$$

We consider two linear dynamic systems

$$
x^{\Delta}(t)=A(t) x(t), t \in \mathbb{T},
$$

and

$$
y^{\Delta}(t)=B(t) y(t), t \in \mathbb{T},
$$


where $A, B \in C_{\mathrm{rd}} \mathcal{R}\left(\mathbb{T}^{\kappa}, M_{n}(\mathbb{R})\right)$.

We consider the adjoint system of (2.1)

$$
x^{\Delta}=(\ominus A)^{*}(t) x, t \in \mathbb{T},
$$

where $A^{*}$ denotes the conjugate transpose of a matrix $A$ and $(\ominus A)(t)=-[I+$ $\mu(t) A(t)]^{-1} A(t)$.

We note that the solution $x(t)$ of $(2.3)$ with the initial point $x\left(t_{0}\right)=x_{0}$ is given by

$$
x(t)=\Phi_{\ominus A^{*}}\left(t, t_{0}\right) x_{0}=\left(\Phi_{A}^{-1}\left(t, t_{0}\right)\right)^{*} x_{0}
$$

and

$$
\Phi_{\ominus A^{*}}^{-1}\left(t, t_{0}\right)=\Phi_{A}^{*}\left(t, t_{0}\right), t \in \mathbb{T}_{t_{0}}
$$

where $\Phi_{A}(t)$ is a fundamental matrix solution for $(2.1)$ and $\Phi\left(t, t_{0}\right) \equiv \Phi(t) \Phi\left(t_{0}\right)^{-1}$ $[15$, Theorem 6.2].

Choi and Koo [8] introduced $u_{\infty}$-similarity on time scales in order to unify (continuous ) $t_{\infty}$-similarity and (discrete) $n_{\infty}$-similarity that preserves the stability properties for linear dynamics systems on time scales.

Definition 2.2. Let $A, B \in C_{\mathrm{rd}} \mathcal{R}\left(\mathbb{T}^{\kappa}, M_{n}(\mathbb{R})\right)$ and $t_{0} \in \mathbb{T}$. An operator $A$ is $u_{\infty}$-similar to an operator $B$ if there exists an absolutely integrable operator $F \in C_{\mathrm{rd}}\left(\mathbb{T}, M_{n}(\mathbb{R})\right)$, i.e., $\int_{t_{0}}^{\infty}|F(t)| \Delta t<\infty$, such that

$$
S^{\Delta}(t)+S^{\sigma}(t) B(t)-A(t) S(t)=F(t), t \in \mathbb{T}^{\kappa},
$$

for both bounded operators $S$ and $S^{-1} \in C_{\mathrm{rd}}^{1}\left(\mathbb{T}^{\kappa}, \mathfrak{M}_{n}(\mathbb{R})\right)$.

We introduce the notions of asymptotic equilibrium and asymptotic equivalence for linear dynamic systems on time scales as the notions for differential systems in [16].

Definition 2.3. System (2.1) is said to have asymptotic equilibrium if there exists a single $\xi \in \mathbb{R}^{n}$ and $r>0$ such that any solution $x\left(t, t_{0}, x_{0}\right)$ of (2.1) with $\left|x_{0}\right|<r$ satisfies

$$
x\left(t, t_{0}, x_{0}\right)=\xi+o(1) \text { as } t \rightarrow \infty, t \in \mathbb{T}_{t_{0}}
$$

and for every $\xi \in \mathbb{R}^{n}$, there exists a solution of (2.1) such that satisfies the above asymptotic relationship.

Definition 2.4. Two systems (2.1) and (2.2) are said to be asymptotically equivalent if, for every solution $x(t)$ of $(2.1)$, there exists a solution $y(t)$ of $(2.2)$ such that

$$
x(t)=y(t)+o(1) \text { as } t \rightarrow \infty, t \in \mathbb{T}_{t_{0}}
$$

and conversely, for every solution $y(t)$ of $(2.2)$, there exists a solution $x(t)$ of (2.1) such that the above asymptotic relation holds. 
Remark 2.5 ([5, Example 4.3]). Note that if system (2.1) has asymptotic equilibrium, then system (2.1) is strongly stable, but the converse does not hold in general. So, the converse holds under a certain condition, i.e., if system (2.1) is strongly stable and $\lim _{t \rightarrow \infty} \Phi_{A}(t)=\Phi_{\infty}$ exists, then system (2.1) has asymptotic equilibrium.

Theorem 2.6 ([8, Theorems 4.3 and 4.9]). For each fixed $\tau \in \mathbb{T}$ the following statements are equivalent:

(i) System (2.1) is stable together with its adjoint system (2.3).

(ii) There exists a positive constant $M$ such that

$$
\left|\Phi_{A}(t, \tau)\right| \leq M \text { and }\left|\Phi_{A}^{-1}(t, \tau)\right| \leq M, t \in \mathbb{T}_{\tau} .
$$

(iii) There exists a positive constant $M$ such that

$$
\left|\Phi_{A}(t, s)\right| \leq M, \quad t, s \in \mathbb{T}_{\tau} .
$$

(iv) System (2.1) is kinematically similar to $x^{\Delta}=0$ on $\mathbb{T}_{\tau}$.

Theorem 2.7. System (2.1) has asymptotic equilibrium if and only if

$$
\lim _{t \rightarrow \infty} \Phi_{A}(t)
$$

exists and is invertible, where $\Phi_{A}(t)$ is a fundamental matrix solution of system (2.1).

Proof. Suppose that system (2.1) has asymptotic equilibrium. Then it is easy to show the existence of $\lim _{t \rightarrow \infty} \Phi_{A}\left(t, t_{0}\right)=\lim _{t \rightarrow \infty} \Phi_{A}(t) \Phi_{A}\left(t_{0}\right)^{-1}=\Phi_{\infty}$.

Let $E_{i}=(0, \ldots, 1, \ldots, 0)^{T}$ be the $i$-th unit vector in $\mathbb{R}^{n}$ for each $i=$ $1,2, \ldots, n$. Then there exist the solutions $x\left(t, t_{0}, x_{0 i}\right)$ of $(2.1)$ such that

$$
\lim _{t \rightarrow \infty} x\left(t, t_{0}, x_{0 i}\right)=\lim _{t \rightarrow \infty} \Phi_{A}\left(t, t_{0}\right) x_{0 i}=E_{i}, i=1,2, \ldots, n .
$$

It follows that

$$
\lim _{t \rightarrow \infty} \Phi_{A}\left(t, t_{0}\right)\left[x_{01} \cdots x_{0 n}\right]=\Phi_{\infty}\left[x_{01} \cdots x_{0 n}\right]=I,
$$

where $I$ is the identity matrix. Thus $\Phi_{\infty}$ is invertible.

We easily see that the converse holds. This completes the proof.

We give an example to illustrate Theorem 2.7.

Example 2.8 ([8, Example 4.17]). Let $t_{0} \in \mathbb{T}$. We consider the linear dynamic system

$$
x^{\Delta}=A(t) x=\left(\begin{array}{cc}
\frac{-e^{-t}}{2+e^{-t}} & 0 \\
0 & 0
\end{array}\right) x, x\left(t_{0}\right)=x_{0}, t \in \mathbb{T}_{t_{0}}
$$

where $A(t)=\left(\begin{array}{cc}\frac{-e^{-t}}{2+e^{-t}} & 0 \\ 0 & 0\end{array}\right) \in C_{\mathrm{rd}} \mathcal{R}\left(\mathbb{T}, M_{2}(\mathbb{R})\right)$. If $\mu(t)$ is a nonnegative constant satisfying $\mu(t)<2 e^{t}+1$ for each fixed $t \in \mathbb{T}_{t_{0}}$, then system (2.5) has asymptotic equilibrium. 
Proof. A fundamental matrix solution $\Phi_{A}\left(t, t_{0}\right)$ of $(2.5)$ is given by

$$
\Phi_{A}\left(t, t_{0}\right)=\left(\begin{array}{cc}
e_{p}\left(t, t_{0}\right) & 0 \\
0 & 1
\end{array}\right)
$$

where $p(t)=\frac{-e^{-t}}{2+e^{-t}}$ and $e_{p}\left(t, t_{0}\right)=\exp \int_{t_{0}}^{t} \frac{1}{\mu(\tau)} \log (1+\mu(\tau) p(\tau)) \Delta \tau$. It follows that

$$
\begin{aligned}
0<e_{p}\left(t, t_{0}\right) & =\exp \int_{t_{0}}^{t} \frac{1}{\mu(\tau)} \log (1+\mu(\tau) p(\tau)) \Delta \tau \\
& \leq \exp \int_{t_{0}}^{t} \frac{1}{\mu(\tau)} \log (1+\mu(\tau)|p(\tau)|) \Delta \tau \\
& \leq \exp \int_{t_{0}}^{t} \frac{e^{-\tau}}{2+e^{-\tau}} \Delta \tau\left(t \in \mathbb{T}_{t_{0}}\right) \\
& \leq C
\end{aligned}
$$

where $C$ is a some positive constant. We see that $e_{p}\left(t, t_{0}\right)$ and $\frac{1}{e_{p}\left(t, t_{0}\right)}$ are bounded for each $t \in \mathbb{T}_{t_{0}}$. Thus we have

$$
\begin{aligned}
\left|\Phi_{A}\left(t, t_{0}\right)\right| & =\left|\left(\begin{array}{cc}
e_{p}\left(t, t_{0}\right) & 0 \\
0 & 1
\end{array}\right)\right| \leq M, \\
\left|\Phi_{A}^{-1}\left(t, t_{0}\right)\right| & =\left|\left(\begin{array}{cc}
1 & 0 \\
0 & \frac{1}{e_{p}\left(t, t_{0}\right)}
\end{array}\right)\right| \leq M, t \in \mathbb{T}_{t_{0}},
\end{aligned}
$$

where $M$ is a positive constant. Thus system (2.5) is strongly stable by Theorem 2.6.

Furthermore, we note that $e_{p}\left(t, t_{0}\right)$ is also nondecreasing on $\mathbb{T}_{t_{0}}$ since the function $1+\mu(t) p(t)$ is positive and nondecreasing on $\mathbb{T}_{t_{0}}$ from the condition of $\mu(t)$. Thus $\lim _{t \rightarrow \infty} e_{p}\left(t, t_{0}\right)$ exists and is a nonzero constant. In fact, this implies that $\lim _{t \rightarrow \infty} \Phi_{A}\left(t, t_{0}\right)$ exists and is invertible. Hence it follows from Theorem 2.7 that (2.5) has asymptotic equilibrium. This completes the proof.

Remark 2.9 ([8, Remark 4.18]). We give some remarks about Example 2.8:

(1) If $\mathbb{T}=\mathbb{R}$, then a fundamental matrix solution $\Phi_{A}(t, 0)$ of linear differential system $x^{\Delta}=x^{\prime}=A(t) x$ is given by

$$
\Phi_{A}(t, 0)=\left(\begin{array}{cc}
\frac{2+e^{-t}}{3} & 0 \\
0 & 1
\end{array}\right), t \in \mathbb{R}_{+} .
$$

Thus we easily see that

$$
\lim _{t \rightarrow \infty} \Phi_{A}(t, 0)=\left(\begin{array}{cc}
\lim _{t \rightarrow \infty}\left(\frac{2+e^{-t}}{3}\right) & 0 \\
0 & 1
\end{array}\right)=\left(\begin{array}{ll}
\frac{2}{3} & 0 \\
0 & 1
\end{array}\right) .
$$


(2) If $\mathbb{T}=h \mathbb{Z}$ with the positive constant $\mu(t)=h<2 e^{t}+1$ for each $t \in h \mathbb{Z}$, then a fundamental matrix solution $\Phi_{A}(t)$ of linear difference system $x^{\Delta}=x(t+h)-x(t)=A(t) x$ is given by

$$
\Phi_{A}(t, 0)=\left(\begin{array}{cc}
\prod_{\tau=0}^{t-h}\left(1-\frac{e^{-\tau}}{2+e^{-\tau}} h\right)^{\frac{1}{h}} & 0 \\
0 & 1
\end{array}\right), t \in h \mathbb{Z} .
$$

We note that $\prod_{\tau=0}^{t-h}\left(1-\frac{e^{-\tau}}{2+e^{-\tau}} h\right)^{\frac{1}{h}}$ is bounded and nondecreasing for each $t \in h \mathbb{Z}$ since $\left(1-\frac{e^{-\tau}}{2+e^{-\tau}} h\right)^{\frac{1}{h}}$ is positive and nondecreasing for each $\tau \in h \mathbb{Z}$. Thus we have the invertible matrix $\Phi_{\infty}$ given by

$$
\lim _{t \rightarrow \infty} \Phi_{A}(t, 0)=\lim _{t \rightarrow \infty}\left(\begin{array}{cc}
\prod_{\tau=0}^{t-h}\left(1-\frac{e^{-\tau}}{2+e^{-\tau}} h\right)^{\frac{1}{h}} & 0 \\
0 & 1
\end{array}\right)=\left(\begin{array}{cc}
a_{\infty} & 0 \\
0 & 1
\end{array}\right) \equiv \Phi_{\infty},
$$

where $\lim _{t \rightarrow \infty} \prod_{\tau=0}^{t-h}\left(1-\frac{e^{-\tau}}{2+e^{-\tau}} h\right)^{\frac{1}{h}}=a_{\infty}$ is positive.

(3) In particular, if $\mathbb{T}=\mathbb{Z}$, then a fundamental matrix solution $\Phi_{A}(t, 0)$ of $\Delta x=x(t+1)-x(t)=A(t) x$ is given by

$$
\Phi_{A}(t, 0)=\left(\begin{array}{cc}
\prod_{\tau=0}^{t-1}\left(1-\frac{e^{-\tau}}{2+e^{-\tau}}\right) & 0 \\
0 & 1
\end{array}\right), t \in \mathbb{Z}_{+} .
$$

Note that the invertible matrix $\Phi_{\infty}$ is given by

$$
\lim _{t \rightarrow \infty} \Phi_{A}(t, 0)=\lim _{t \rightarrow \infty}\left(\begin{array}{cc}
\prod_{\tau=0}^{t-1}\left(1-\frac{e^{-\tau}}{2+e^{-\tau}}\right) & 0 \\
0 & 1
\end{array}\right)=\left(\begin{array}{cc}
b_{\infty} & 0 \\
0 & 1
\end{array}\right) \equiv \Phi_{\infty}
$$

where $\lim _{t \rightarrow \infty} \prod_{\tau=0}^{t-1}\left(1-\frac{e^{-\tau}}{2+e^{-\tau}}\right)=b_{\infty}$ is positive for each $t \in \mathbb{Z}_{+}$.

Lemma 2.10 ([13, Corollary 2.4]). Let $A \in \mathrm{C}_{r d} R\left(\mathbb{T}^{\kappa}, M_{n}(\mathbb{R})\right)$ be an $n \times n$ matrix-valued function and assume that $\Phi_{A}(t)$ is a solution of $X^{\Delta}=A(t) X$. Then $\Phi_{A}(t)$ satisfies Liouville's formula

$$
\operatorname{det} \Phi_{A}(t)=e_{q}\left(t, t_{0}\right) \operatorname{det} \Phi_{A}\left(t_{0}\right), t \in \mathbb{T},
$$

where $q(t)=\lambda_{1} \oplus \lambda_{2} \oplus \cdots \oplus \lambda_{n}$ and each $\lambda_{i}, 1 \leq i \leq n$, is the eigenvalue of $A(t)$. Here $\oplus$ is defined by $a \oplus b=a+b+\mu(t) a b$.

Remark 2.11 ([3, Theorem 5.28]). When $\mathbb{T}=\mathbb{R}$, we have

$$
\begin{aligned}
q(t) & =\lambda_{1} \oplus \lambda_{2} \oplus \cdots \oplus \lambda_{n} \\
& =\lambda_{1}+\lambda_{2}+\cdots+\lambda_{n}=\operatorname{tr} A(t) .
\end{aligned}
$$

Also, if $A(t)$ is a regressive $2 \times 2$ matrix-valued function, then

$$
q(t)=\operatorname{tr} A(t)+\mu(t) \operatorname{det} A(t) .
$$

Theorem 2.12. If system (2.1) has asymptotic equilibrium, then

$$
\lim _{t \rightarrow \infty} e_{q}\left(t, t_{0}\right)
$$

exists. Here $q(t)=\lambda_{1} \oplus \lambda_{2} \oplus \cdots \oplus \lambda_{n}$ and each $\lambda_{i}, 1 \leq i \leq n$, is the eigenvalue of $A(t)$. 
Proof. It follows from Lemma 2.10 that $\Phi_{A}(t)$ satisfies the Liouville's formula:

$$
\operatorname{det} \Phi_{A}(t)=\operatorname{det} \Phi_{A}\left(t_{0}\right) e_{q}\left(t, t_{0}\right), t \in \mathbb{T}_{t_{0}} .
$$

Thus, we have

$$
\begin{aligned}
0 \neq \operatorname{det} \Phi_{\infty} & =\lim _{t \rightarrow \infty} \operatorname{det} \Phi_{A}(t) \\
& =\operatorname{det} \Phi_{A}\left(t_{0}\right) \lim _{t \rightarrow \infty} e_{q}\left(t, t_{0}\right) .
\end{aligned}
$$

Hence $\lim _{t \rightarrow \infty} e_{q}\left(t, t_{0}\right)$ exists. The proof is complete.

We can obtain the following result as the special case of Theorem 2.12.

Corollary 2.13 ([10, Theorem 3.3]). Suppose that system (2.1) has asymptotic equilibrium and $\mathbb{T}=\mathbb{R}$. Then

$$
\lim _{t \rightarrow \infty} \int_{t_{0}}^{t} \operatorname{tr} A(s) d s
$$

exists.

Proof. It follows from Remark 2.11 that we have

$$
\lim _{t \rightarrow \infty} e_{p}\left(t, t_{0}\right)=\exp \left(\lim _{t \rightarrow \infty} \int_{t_{0}}^{t} \operatorname{tr} A(s) d s\right) .
$$

From Theorem 2.12, $\lim _{t \rightarrow \infty} \int_{t_{0}}^{t} \operatorname{tr} A(s) d s$ exists. This completes the proof.

The following example shows that the converse of Corollary 2.13 does not hold in general:

Example 2.14. Let $\mathbb{T}=\mathbb{R}$ and consider the linear dynamic system

$$
x^{\Delta}(t)=x^{\prime}(t)=\left(\begin{array}{cc}
t & 0 \\
0 & -t
\end{array}\right) x, t \in \mathbb{R}_{+},
$$

where $A(t)=\left(\begin{array}{cc}t & 0 \\ 0 & -t\end{array}\right)$. A fundamental matrix solution $\Phi_{A}(t)$ of system $(2.8)$ is given by

$$
\Phi_{A}(t)=\left(\begin{array}{cc}
e^{\frac{t^{2}}{2}} & 0 \\
0 & e^{-\frac{t^{2}}{2}}
\end{array}\right), t \in \mathbb{R}_{+} .
$$

Then we easily see that $\lim _{t \rightarrow \infty} \int_{t_{0}}^{t} \operatorname{tr} A(s) d s=0$ but $\lim _{t \rightarrow \infty} \Phi_{A}(t)$ does not exist.

Choi and Koo [8] obtained the following result about the stable stability that is preserved by the notion of $u_{\infty}$-similarity.

Lemma 2.15 ([8, Theorem 4.13]). Assume that systems (2.1) and (2.2) are $u_{\infty}$-similar. Then system (2.1) is strongly stable if and only if system (2.2) is also strongly stable. 
We can obtain the following result that the property of asymptotic equilibra for linear dynamic systems on time scales is preserved by the notion of $u_{\infty}$ similarity.

Theorem 2.16. Suppose that $A$ and $B$ are $u_{\infty}$-similar with

$$
\lim _{t \rightarrow \infty} S(t)=S_{\infty}
$$

Then system (2.1) has asymptotic equilibrium if and only if system (2.2) also has asymptotic equilibrium.

Proof. Suppose that system (2.1) has asymptotic equilibrium. Then (2.2) is strongly stable by Lemma 2.15. Also, the assumption on $S(t)$ implies that $\lim _{t \rightarrow \infty} S(t)=S_{\infty}$ is invertible and $\lim _{t \rightarrow \infty} S^{-1}(t)=S_{\infty}^{-1}$. Since $\int_{t_{0}}^{\infty}|F(t)| \Delta t$ $<\infty$, and $\Phi_{A}(t, s)$ and $\Phi_{B}\left(t, t_{0}\right)$ are bounded, we easily see from the Cauchy property that

$$
\lim _{t \rightarrow \infty} \int_{t_{0}}^{t} \Phi_{A}(t, s) F(s) \Phi_{B}\left(s, t_{0}\right) \Delta s
$$

exists. It follows that $\lim _{t \rightarrow \infty} \Phi_{B}(t)=\Phi_{\infty}$ exists and is also invertible. Therefore (2.2) has asymptotic equilibrium by Theorem 2.7.

The converse holds by the same manner. This completes the proof.

Remark 2.17. Continuous version and discrete version of Theorem 2.16 were presented in [10, Theorem 3.6] and [5, Theorem 4.6], respectively.

Now, we can obtain the following result about asymptotic equivalence by using the concepts of $u_{\infty}$-similarity and asymptotic equilibrium.

Theorem 2.18. Assume that

(i) there exists a positive constant $\alpha$ with $\left|\operatorname{det}\left(\Phi_{A}(t)\right)\right|>\alpha>0$ for each $t \in \mathbb{T}_{t_{0}}$ and $\lim _{t \rightarrow \infty} \Phi_{A}(t)=\Phi_{\infty}$ exists

(ii) $A$ and $B$ are $u_{\infty}$-similar with $\lim _{t \rightarrow \infty} S(t)=S_{\infty}$.

Then two systems (2.1) and (2.2) are asymptotically equivalent.

Proof. We easily see that (2.1) has asymptotic equilibrium by the fact that $\left|\operatorname{det}\left(\Phi_{\infty}\right)\right| \geq \alpha>0$ and Theorem 2.7. It follows from the assumption (ii) and Theorem 2.16 that $(2.2)$ has asymptotic equilibrium. Let $x\left(t, t_{0}, x_{0}\right)$ be any solution of $(2.1)$. Then $\lim _{t \rightarrow \infty} x(t)=x_{\infty}$ exists. For each $x_{\infty} \in \mathbb{R}^{n}$, the condition on asymptotic equilibrium for (2.2) implies that there exists a solution $y(t)=y\left(t, t_{0}, y_{0}\right)$ of $(2.2)$ such that $\lim _{t \rightarrow \infty} y(t)=x_{\infty}$. This implies that

$$
y(t)=x(t)+o(1) \text { as } t \rightarrow \infty .
$$

By the same manner, we can obtain the converse asymptotic relationship.

Remark 2.19. Continuous version and discrete version of Theorem 2.18 were presented in [10, Theorem 3.7] and [5, Theorem 4.7], respectively. 
Finally, we study the asymptotic equivalence between homogeneous and nonhomogeneous linear dynamic systems on time scales by means of asymptotic equilibrium of homogeneous linear dynamic systems. So we consider the perturbed system of $(2.1)$

$$
y^{\Delta}(t)=A(t) y(t)+g(t), t \in \mathbb{T}_{t_{0}},
$$

where $A \in C_{\mathrm{rd}} \mathcal{R}\left(\mathbb{T}, M_{n}(\mathbb{R})\right)$ and $g: \mathbb{T}_{t_{0}} \rightarrow \mathbb{R}^{n}$ is an rd-continuous function.

Lemma 2.20. Assume that system (2.1) has asymptotic equilibrium and the perturbed term $g$ in (2.9) is absolutely integrable on $\mathbb{T}_{t_{0}}$, i.e.,

$$
\int_{t_{0}}^{\infty}|g(s)| \Delta s<\infty
$$

Then system (2.9) also has asymptotic equilibrium.

Proof. It follows that any solution $y(t)=y\left(t, t_{0}, y_{0}\right)$ of $(2.9)$ is given by

$$
y(t)=\Phi_{A}\left(t, t_{0}\right) y_{0}+\Phi_{A}\left(t, t_{0}\right) \int_{t_{0}}^{t} \Phi_{A}^{-1}\left(s, t_{0}\right) g(s) \Delta s,
$$

where $\Phi_{A}(t)$ is a fundamental matrix solution of (2.1) and

$$
\Phi_{A}\left(t, t_{0}\right)=\Phi_{A}(t) \Phi_{A}^{-1}\left(t_{0}\right) .
$$

Putting $p(t)=\int_{t_{0}}^{t} \Phi_{A}^{-1}\left(s, t_{0}\right) g(s) \Delta s$, we easily see that $p(t)$ has a finite limit as $t \rightarrow \infty$ because $\int_{t_{0}}^{\infty}|g(s)| \Delta s<\infty$ and $\Phi_{A}^{-1}(t)$ is bounded for each $t \in \mathbb{T}_{t_{0}}$. Thus $y(t)$ converges to a vector $\xi \in \mathbb{R}^{n}$ as $t \rightarrow \infty$.

Conversely, let $\xi$ be any vector in $\mathbb{R}^{n}$. Then there exists a solution $y(t)=$ $y\left(t, t_{0}, y_{0}\right)$ of $(2.9)$ with the initial point $y_{0}=\Phi_{\infty}^{-1} \xi-p_{\infty}$ such that

$$
\begin{aligned}
\lim _{t \rightarrow \infty} y(t) & =\lim _{t \rightarrow \infty}\left[\Phi_{A}\left(t, t_{0}\right) y_{0}+\Phi_{A}\left(t, t_{0}\right) \int_{t_{0}}^{t} \Phi_{A}^{-1}\left(s, t_{0}\right) g(s) \Delta s\right] \\
& =\Phi_{\infty}\left[y_{0}+p_{\infty}\right] \\
& =\Phi_{\infty}\left[\Phi_{\infty}^{-1} \xi-p_{\infty}+p_{\infty}\right] \\
& =\xi
\end{aligned}
$$

where $\lim _{t \rightarrow \infty} p(t)=p_{\infty}$ and $\lim _{t \rightarrow \infty} \Phi_{A}\left(t, t_{0}\right)=\Phi_{\infty}$ is invertible. This completes the proof.

As a consequence of Lemma 2.20 we easily obtain the following result.

Theorem 2.21. Suppose that system (2.1) has asymptotic equilibrium and $\int_{t_{0}}^{\infty}|g(s)| \Delta s<\infty$ for each fixed $t_{0} \in \mathbb{T}$. Then two systems (2.1) and (2.9) are asymptotically equivalent.

Proof. Let $x(t)$ be any solution of (2.1). Then we have $\lim _{t \rightarrow \infty} x(t)=x_{\infty}$ since (2.1) has asymptotic equilibrium. Setting $y_{0}=\Phi_{\infty}^{-1} x_{\infty}-p_{\infty}$ as in Lemma 2.20, there exists a solution $y\left(t, t_{0}, y_{0}\right)$ of $(2.9)$ such that

$$
\lim _{t \rightarrow \infty}[y(t)-x(t)]=\Phi_{\infty}\left[y_{0}+p_{\infty}\right]-x_{\infty}
$$




$$
\begin{aligned}
& =\Phi_{\infty}\left[\Phi_{\infty}^{-1} x_{\infty}-p_{\infty}+p_{\infty}\right]-x_{\infty} \\
& =0 .
\end{aligned}
$$

Conversely, we easily see that the asymptotic relationship also holds by setting $x_{0}=y_{0}+p_{\infty}$. This completes the proof.

Remark 2.22. Continuous version and discrete version of Theorem 2.21 were presented in [10, Theorem 3.9] and [5, Theorem 4.9], respectively.

We give an example to illustrate Theorem 2.21.

Example 2.23. We consider homogeneous dynamic system

$$
x^{\Delta}(t)=A(t) x(t)=\left(\begin{array}{cc}
\frac{-e^{-t}}{2+e^{-t}} & 0 \\
0 & 0
\end{array}\right) x(t), t \in \mathbb{T}_{t_{0}},
$$

and nonhomogeneous dynamic system

$$
y^{\Delta}(t)=A(t) y(t)+g(t)=\left(\begin{array}{cc}
\frac{-e^{-t}}{2+e^{-t}} & 0 \\
0 & 0
\end{array}\right) y(t)+\left(\begin{array}{l}
e^{-t} \\
e^{-t}
\end{array}\right), t \in \mathbb{T}_{t_{0}} .
$$

Assume that $\mu(t)$ is a nonnegative constant satisfying $\mu(t)<2 e^{t}+1$ for each $t \in \mathbb{T}_{t_{0}}$. Then it follows from the simple calculation that two systems $(2.10)$ and (2.11) are asymptotically equivalent by Example 2.8 and Theorem 2.21.

Acknowledgment. This work was supported by Basic Science Research Program through the National Research Foundation of Korea(NRF) funded by the Ministry of Education(NRF-2013R1A1A2007585).

\section{References}

[1] B. Aulbach and S. Hilger, A unified approach to continuous and discrete dynamics, in "Qualitative Theory of Differential Equations"(Szeged, 1988), 37-56, Colloq. Math. Soc. János Bolyai, 53, North Holland, Amsterdam, 1990.

[2] — Linear dynamic processes with inhomogeneous time scale, in "Nonlinear Dynamics and Quantum Dynamical Systems"(Gaussig, 1990), 9-20, Math. Res. 59, Akademie-Verlag, Berlin, 1990.

[3] M. Bohner and A. Peterson, Dynamic Equations on Time Scales: An Introduction with Applications, Birkhäuser, Boston, 2001.

[4] _ Advances in Dynamic Equations on Time Scales, Birkhäuser, Boston, 2003.

[5] S. K. Choi, Y. H. Goo, and N. J. Koo, Variationally asymptotically stable difference systems, Adv. Difference Equ. 2007 (2007), Article ID 35378, 21 pp.

[6] S. K. Choi, Y. H. Goo, and N. Koo, h-Stability of dynamic equations on time scales with nonregressivity, Abstr. Appl. Anal. 2008 (2008), Article ID 632473, 13 pp.

[7] S. K. Choi and N. J. Koo, Variationally stable difference systems by $n_{\infty}$-similarity, J. Math. Anal. Appl. 249 (2000), no. 2, 553-568.

[8] S. K. Choi and N. Koo, On the stability of linear dynamic systems on time scales, J. Difference Equ. Appl. 15 (2009), no. 2, 167-183.

[9] S. K. Choi, N. J. Koo, and S. Dontha, Asymptotic property in variation for nonlinear differential systems, Appl. Math. Lett. 18 (2005), no. 1, 117-126.

[10] S. K. Choi, N. J. Koo, and D. M. Im, Asymptotic equivalence between linear differential systems, Bull. Korean Math. Soc. 42 (2005), no. 4, 691-701. 
[11] S. K. Choi, N. J. Koo, and K. Lee, Asymptotic equivalence for linear differential systems, Commun. Korean Math. Soc. 26 (2011), no. 1, 37-49.

[12] R. Conti, Sulla $t_{\infty}$-similitudine tra matrici e la stabilità dei sistemi differenziali lineari, Atti. Acc. Naz. Lincei, Rend. Cl. Fis. Mat. Nat. 49 (1955), 247-250.

[13] V. Cormani, Liouville's formula on time scales, Dynam. Systems Appl. 12 (2003), no. $1-2,79-86$.

[14] I. Gohberg, M. A. Kaashoek, and J. Kos, Classification of linear time-varying difference equations under kinematic similarity, Integral Equations Operator Theory 25 (1996), no. $4,445-480$.

[15] S. Hilger, Analysis on measure chains - a unified approach to continous and discrete calculus, Results Math. 18 (1990), no. 1-2, 18-56.

[16] V. Lakshmikantham and S. Leela, Differential and Integral Inequalites with Theory and Applications, Academic Press, New York and London, 1969.

[17] L. Markus, Continuous matrices and the stability of differential systems, Math. Z. 62 (1955), 310-319.

[18] W. F. Trench, On $t_{\infty}$ quasi-similarity of linear systems, Ann. Mat. Pura Appl. 142 (1985), 293-302.

[19] L Linear asymptotic equilibrium and uniform, exponential, and strict stability of linear difference systems, Comput. Math. Appl. 36 (1998), no. 10-12, 261-267.

Sung Kyu ChOI

Department of Mathematics

Chungnam National University

DAEJEON 305-764, KOREA

E-mail address: sgchoi@cnu.ac.kr

NAMJIP KOO

Department of Mathematics

Chungnam National University

DAEJeON 305-764, KoREA

E-mail address: njkoo@cnu.ac.kr 